\title{
BMJ open Prediction of initiation and cessation of breastfeeding from late pregnancy to 16 weeks: the Feeding Your Baby (FYB) cohort study
}

\author{
Peter T Donnan, ${ }^{1}$ Janet Dalzell, ${ }^{2}$ Andrew Symon, ${ }^{3}$ Petra Rauchhaus, ${ }^{1}$ \\ Ewa Monteith-Hodge, ${ }^{3}$ Gillian Kellett, ${ }^{3}$ Jeremy C Wyatt, ${ }^{4}$ Heather M Whitford ${ }^{3}$
}

To cite: Donnan PT, Dalzell J, Symon A, et al. Prediction of initiation and cessation of breastfeeding from late pregnancy to 16 weeks: the Feeding Your Baby (FYB) cohort study. BMJ Open 2013;3:e003274.

doi:10.1136/bmjopen-2013003274

- Prepublication history and additional material for this paper is available online. To view these files please visit the journal online (http://dx.doi.org/10.1136/ bmjopen-2013-003274).

Received 21 May 2013 Revised 26 June 2013 Accepted 28 June 2013

${ }^{1}$ Dundee Epidemiology and Biostatistics Unit, Division of Population Health Sciences, Medical Research Institute, University of Dundee, Dundee, Scotland, UK ${ }^{2}$ Directorate of Public Health, NHS, Tayside, Scotland, UK ${ }^{3}$ School of Nursing and Midwifery, University of Dundee, Dundee, Scotland, UK

${ }^{4}$ Leeds Institute of Health Sciences, University of Leeds, Leeds, UK

Correspondence to Dr Heather M Whitford; h.m.whitford@dundee.ac.uk

\section{ABSTRACT}

Objective: To derive prediction models for both initiation and cessation of breastfeeding using demographic, psychological and obstetric variables.

Design: A prospective cohort study.

Setting: Women delivering at Ninewells Hospital, Dundee, UK.

Data sources: Demographic data and psychological measures were obtained during pregnancy by questionnaire. Birth details, feeding method at birth and at hospital discharge were obtained from the Ninewells hospital database, Dundee, UK. Breastfeeding women were followed up by text messages every 2 weeks until 16 weeks or until breastfeeding was discontinued to ascertain feeding method and feeding intentions.

Participants: Pregnant women over 30 weeks gestation aged 16 years and above, living in Dundee, booked to deliver at Ninewells Hospital, Dundee, and able to speak English.

Main outcome measure: Initiation and cessation of breastfeeding.

Results: From the total cohort of women at delivery $(\mathrm{n}=344) 68 \%(95 \% \mathrm{Cl} 63 \%$ to $73 \%)$ of women had started breastfeeding at discharge. Significant predictors of initiating breastfeeding were older age, parity, greater intention to breastfeed from a Theory of Planned Behaviour (TPB)-based questionnaire, higher lowa Infant Feeding Assessment Scale (IIFAS) score as well as living with a husband or partner. For the final model, the AUROC was 0.967. For those who initiated breastfeeding $(n=233)$, the strongest predictors of stopping were low intention to breastfeed from TPB, low IIFAS score and non-managerial/professional occupations.

Conclusions: The findings from this study will be used to inform the protocol for an intervention study to encourage and support prolonged breastfeeding as intentions appear to be a key intervention focus for initiation. The predictive models could be used to identify women at high risk of not initiating and also women at high risk of stopping for interventions to improve the longevity of breastfeeding.

\section{ARTICLE SUMMARY}

Article focus

- To identify antenatal factors which predict women who will initiate breastfeeding.

- Assess the critical time points for the discontinuation of breastfeeding.

- To identify the key antenatal and postnatal attributes and beliefs associated with continuation/ cessation and develop predictive models.

\section{Key messages}

- Comprehensive assessment of intentions and breastfeeding via novel SMS text messaging facilitated the accurate prediction of breastfeeding initiation and cessation.

- Psychological factors as well as previous experience were shown to be important predictors of cessation before 16 weeks in predictive algorithms indicating the potential for early intervention.

- These findings challenge the current interpretation of the UNICEF guidelines and suggest that a full discussion about infant feeding options in the antenatal period, including asking about intentions, could be used to identify women at risk of early cessation of breastfeeding.

Strengths and limitations of this study

- A key strength was the accurate, validated, realtime and efficient measurement of method of infant feeding through SMS messaging. The study incorporated intentions and psychological factors based on the Theory of Planned Behaviour as predictors of initiation and cessation. This allowed the development of predictive algorithms and could allow the development and trialling of targeted interventions. This was based on a relatively large cohort covering the antenatal period to 16 weeks postnatal. One limitation may be the lack of ethnic diversity in the study population which is reflected in the ethnic structure of Tayside. 


\section{INTRODUCTION}

The short-term and long-term health benefits of breastfeeding for both mother and child are well documented. ${ }^{1-4}$ Consequently, the current WHO recommendation is that infants should be exclusively breastfed for the first 6 months. ${ }^{5}$ Most developed countries report that a minority of infants are exclusively breastfeeding at 6 months (40\% the Netherlands; $13 \%$ the USA) and in the UK exclusive breastfeeding continued after 6 months in less than $1 \%{ }^{6}$ There has been some success in the UK in improving the number of women who start breastfeeding: initiation rates of breastfeeding rose in Scotland from $63 \%$ in 2000 to $74 \%$ in $2010 .{ }^{7}$ However, targets to improve the rate of exclusive feeding at 6-8 weeks have proved to be more challenging. The Scottish Government aimed to increase exclusive breastfeeding at $6-8$ weeks over a 4 -year period to $33.3 \%$ by $2010 / 2011^{8}$; however, in $2010 / 2011$, the rate remained unchanged at $26.5 \% .^{9}$ Given the rapid decline in breastfeeding in the immediate postnatal period, the failure to meet government targets and follow WHO recommendations, more detailed information about current practices and attitudes and the potential for intervention is required.

Maternal demographics and previous breastfeeding experience are known to be associated with initiation as well as duration of breastfeeding ${ }^{9}{ }^{10}$; however, these variables are not amenable to behavioural change interventions. The measurement of attitudinal factors such as the Iowa Infant Feeding Assessment Scale (IIFAS) ${ }^{11}$ has shown promise as a way of improving the accuracy of prediction of the initiation of breastfeeding behaviour. The IIFAS has been found to predict breastfeeding initiation in a variety of settings including the USA, ${ }^{11}$ Australia, ${ }^{12}$ Scotland, ${ }^{13}{ }^{14}$ Northern Ireland $^{15}$ and Romania. ${ }^{16}$ However, these studies have either only measured feeding at birth, ${ }^{14}$ until discharge from hospital, ${ }^{14}{ }^{15}$ or by retrospective maternal report. ${ }^{16}$ The only study which prospectively followed women over a prolonged period was carried out in an area of high breastfeeding (94\% initiation rate) and was biased by the recruitment of women and measurement of baseline variables in the first 3 days after birth (rather than during pregnancy) by which time attitudes to infant feeding are likely to have been affected by experiences since birth. ${ }^{12}$

Hence, there is little evidence for interventions based on psychological and attitudinal variables to improve breastfeeding outcomes. However, a WHO programme (The Baby Friendly Initiative, BFI) to protect and support the initiation and continuation of breastfeeding by the implementation of evidence-based care in maternity hospitals is well established. ${ }^{17}$ Many hospitals and community settings strive to achieve 'UNICEF Baby Friendly Status' and there is some evidence that BFI accreditation can improve initiation and continuation rates. ${ }^{18-20}$ Guidance from UNICEF for Step 3 of BFI accreditation, in the context of information provision, 'strongly recommends that pregnant women are not merely asked a closed question about how they plan to feed their baby' (Ref. 21, p.13). This is to encourage a more open discussion to take place and to allow women to make a final decision about feeding method after delivery. While the recommendation does not explicitly preclude a discussion about feeding intentions in the antenatal period, the guidelines suggest that the documentation of antenatal feeding intention should be avoided. In practice, this has been interpreted more stringently; intentions are not discussed at all.

Building on past research, we designed an exploratory longitudinal study using mixed methodology, including the use of the IIFAS $^{11}$ and psychological variables guided by the Theory of Planned Behaviour (TPB ${ }^{22}$ captured during the antenatal period, to understand and predict women's initiation and duration of breastfeeding in an area of low breastfeeding initiation. Use of the MRC framework ${ }^{23}$ informed the qualitative and quantitative components of the study enabling us to advance our understanding of women's intentions and attitudes towards infant feeding. The study used SMS text messaging, a novel method of data collection, to follow-up women after delivery. The validity and reliability of the method of SMS text messaging has already been reported elsewhere ${ }^{24}$ as well as some of the qualitative results. ${ }^{25}$

This paper reports the identification of (1) antenatal factors which predict women who will initiate breastfeeding; (2) the critical time points for the discontinuation of breastfeeding and (3) the key antenatal and postnatal attributes and beliefs associated with continuation/ cessation.

From these data, a predictive model was derived to identify those at high risk of stopping breastfeeding. The findings from this study will inform the recruitment protocol and design of an intervention to encourage breastfeeding in a future randomised controlled trial testing the intervention efficacy.

\section{METHODS \\ Design}

A prospective cohort study of the method of infant feeding following delivery.

\section{Participants}

Pregnant women over 30 weeks gestation aged 16 years and above, living in Dundee, booked to deliver at Ninewells Hospital and able to speak English. There were no exclusions based on feeding intention or maternal history. The detailed reasons for exclusion are shown in online supplementary appendix 2.

\section{Measures}

Five data collection points were used.

1. Baseline data-self-completed questionnaire, third trimester of pregnancy Background demographic: 
- Age, cohabitation and residency status, years since leaving school and occupation based on Standard Occupational Classification, ONS, 2010. ${ }^{26}$ Socioeconomic status derived from postcode and corresponding SIMD scores.

Obstetric measures:

- Expected date of delivery

- Parity

- Previous infant feeding.

Psychological measures:

- IIFAS $^{11}$ a 17-item questionnaire with 5-point Likert scale response format from strongly agree to strongly disagree. Scores range from 17 to 85: higher score $=$ more positive attitude to breastfeeding.

- TPB questionnaine study-specific 13-item questionnaire informed by the theories of planned behaviour and self-efficacy ${ }^{22}$ assessed Attitude to breastfeeding (four items), Social norm (two items), Perceived Behavioural Control (PBC; three items) and Intention (four items) each recorded on a 5-point scale (see online supplementary appendix 3).

2. Delivery data obtained through hospital records

- Date of delivery, method of delivery, sex and weight of baby, method of infant feeding recorded at birth and at discharge from the hospital.

Outcome variables

3. Infant feeding collected by validated SMS text messages $^{24}$

Method(s) of infant feeding and future intentions, assessed after hospital discharge every 2 weeks using two text questions until response ' $F$ ' received to SMS1:

- SMS1. 'In the past 2 weeks how have you been feeding your baby?' (Answer options-only breast milk $(\mathrm{O})$, both breast and formula milk (B), only formula milk $(\mathrm{F}))$.

- SMS2. If 'only breast milk' or 'both breast and formula milk'-'For how many more weeks do you plan to give your baby breast milk?

4. Exit data (4 weeks after final SMS message):

- Method of infant feeding at study exit, problems with infant feeding, satisfaction with (breast) feeding support and satisfaction with feeding method(s) using 5-point Likert scale response format.

5. Focus groups and interviews with various subgroups of women.

\section{Procedure}

Women were approached in the last trimester of pregnancy at clinics by a Community Midwife (CM) or a Research Assistant (RA). Consent was obtained for contact details to be passed to the study team in the form of returned postcards; women were given a baseline questionnaire and consent form. These were returned to the study team following a recruitment phone call by the RAs. Study incentives were used to motivate and encourage CMs to recruit.

The hospital database was checked weekly; as participants delivered, their delivery and discharge details were sent to the RAs. Starting from 2 weeks after delivery, RAs used standard web-based messaging tools to contact all participants by text to find out current feeding practices and intentions (figure 1). Web-based messaging services sent automated texts via computer and used a text number for responses. Contact continued by text message every 2 weeks until the baby was 16 weeks old, or until the response ' $F$ ' was received. Women with no mobile phone or who preferred not to receive text messages were contacted by the RA on their home phone.

The 'end' point for gathering text data was 2 weeks after delivery for women who started or who changed to formula feeding before 2 weeks, on discontinuation of any breastfeeding or when the baby was 16 weeks old for the rest. Four weeks after the 'end' point, women were contacted by telephone to gather final data (using an exit phone questionnaire). After the exit interview women were sent a thank-you letter and a $£ 10$ gift voucher.

During the exit interview, participants were invited to take part in a focus group or interview. Results are reported elsewhere.

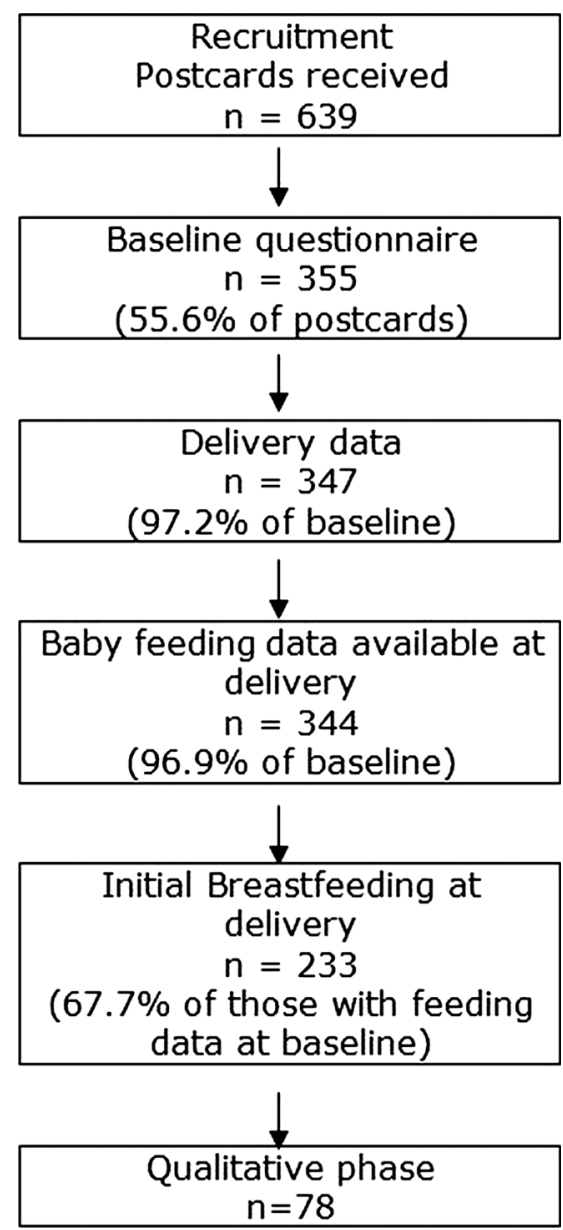

Figure 1 Flow chart of participant recruitment. 


\section{Statistical analysis}

Data were analysed using SAS V.9.2. Descriptive data are presented as \% (frequency) for categorical variables, and mean (95\% CI) for continuous variables.

The total IIFAS score and the subscores for the TPB variables were calculated from the questionnaires. Non-normally distributed variables were converted to categorical variables when there was no viable transformation.

Baseline data were tested for correlations with the duration of breastfeeding. Analysis of variance and $\chi^{2}$ tests were performed to test for significant associations of baseline variables with the duration of breastfeeding and intention to breastfeed, and to examine differences between groups.

The reliability of the text message responses (method of feeding) was checked by repeat-texting a random subset of 50 participants the next day. Validity was checked by telephone contact with a random subset of 50 participants on the same day as their text response and asking them the same questions verbally and by comparing with data collected by the health visitor. The results, previously reported, demonstrated excellent reliability and validity. ${ }^{24}$

Logistic regression modelling was implemented to assess predictors of initiating breastfeeding and the results expressed as relative risks and their 95\% CIs.

For those who initiated breastfeeding, univariate associations between the duration of any and exclusive breastfeeding with baseline variables were performed using the logrank test for each of the baseline variables. Variables with a univariate significance level of at least 0.3 were chosen for potential inclusion in model building.

Cox Proportional Hazards models were then built for all combinations of variables, utilising both a forward and stepwise selection model including all variables. Models were then assessed for goodness of fit using AIC and the best-fit model was chosen. These models were utilised to predict the outcome of any breastfeeding and exclusive breastfeeding.

Model performance was assessed by the estimation of c-statistic, a measure of discrimination as well as the Integrated Discrimination Improvement (IDI) ${ }^{27}$ to demonstrate the most important variables determining discrimination, utilising the SAS macro \%rocplus (http://mayo research.mayo.edu/mayo/research/biostat/sasmacros.cfm). Assessment of calibration was also carried out using methods suitable for censored data. Analyses were implemented in SPSS (V.18) and SAS V.9.2 (SAS Institute, Cary, North Carolina, USA).

\section{Sample size, recruitment and attrition}

The study aimed to recruit 350 women over an 8-month period, giving a recruitment rate of $35 \%$. Of these, approximately $224(64 \%)$ would start breastfeeding (local Maternity Database figures from 2007), and 133 $(38 \%)$ will still be breastfeeding at $6-8$ weeks. ${ }^{9}$ In considering predictors of maintaining breastfeeding at
6-8 weeks from birth, and approximately 130 events, there would be $80 \%$ power to detect HR $\geq 1.6$ in a Cox regression model.

Between November 2009 and June 2010, a total of 639 postcards were received by the study team. From these, 355 women fully consented and were included in the study $(55.6 \%$ of postcards received), which exceeded our target of 350 women (figure 1 and full details in online supplementary appendix 2). The SIMD profile of women who consented broadly tracked the profile of all women who delivered in Dundee in 2009. A total of 292 women were followed up to the exit questionnaire (82.3\% of consented women). Some of this follow-up was protracted due to difficulties in contacting several participants.

At exit, 152 women were asked about participating in a focus group or interview and 138 expressed an interest $(91 \%)$. Of these, 38 took part in one of seven focus groups and 40 were interviewed individually (78 in total, $56 \%$ of those interested, $22 \%$ of total sample). The results of the qualitative analysis are reported elsewhere. ${ }^{25}$

\section{SMS messages for the collection of data about feeding method}

To manage the high number of automated SMS messages, a computer schedule was created for the study (figure 2). A total of 2738 text message responses were received via this automated SMS message scheduler. Data from 42 women were gathered by phone call on 114 occasions when the SMS system was unavailable. The SMS messaging service package incurred a small cost to participants: some participants may have been unable to respond if they had no credit on their phone. Two women were contacted on their home phone only: one had no mobile phone, while the other preferred not to receive text messages.

\section{RESULTS}

\section{Baseline characteristics}

Three hundred and fifty-five women were included in the study at baseline. Of these, $344(96.9 \%)$ had information on feeding status at delivery and prediction of initiating breastfeeding was based on this cohort (table 1). Baseline psychological measures (IIFAS score and TPB) are included in table 1.

\section{Prediction of initiating breastfeeding}

At delivery, $67.7 \%$ (95\% CI $62.8 \%$ to $72.6 \%$ ) of women had started breastfeeding out of those with feeding data $(n=344)$. Significant independent predictors of initiating breastfeeding were older age, parity, greater intention to breastfeed from the TPB questionnaire, higher IIFAS score as well as living with a husband or partner as shown in table 2. For the final logistic model, the AUROC was 0.982 (95\% CI 0.971 to 0.993$)$ and calibration was good with Hosmer-Lemeshow test of $p=0.354$. A score for the estimation of the probability of initiation 
Figure 2 Schedule of SMS messages.

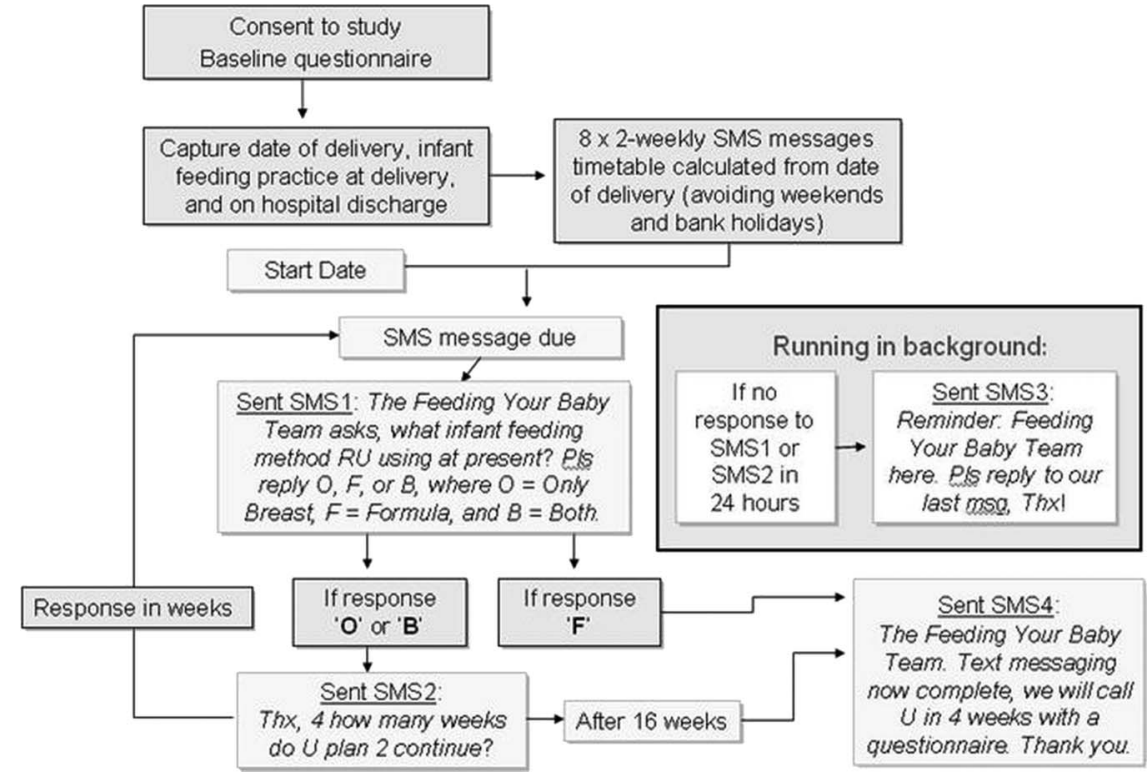

can be easily constructed using this final equation as shown in online supplementary appendix 1 . This score can be utilised as a Clinical Prediction Rule to identify women with low probability of initiating breastfeeding and interventions can be developed that are focused on this group. Estimation of IDI showed that Intention to Breastfeed with an IDI of $0.212(p<0.001)$ was the strongest contributor to discrimination of initiating breastfeeding and entered the model first, followed by the IIFAS score with IDI=0.024 $(\mathrm{p}=0.034)$.

\section{Duration of breastfeeding}

For those with feeding data $(n=344)$, Kaplan-Meier curves were fitted for exclusive breastfeeding (response 'only breast milk' to text question) and any breast milk (response 'both breast and formula milk' to text question) for each of the three subgroups defined by previous breastfeeding and parity. The duration of breastfeeding at various time points was derived (figure 3A,B). These show that parous women who have previous experience of breastfeeding are most likely to start breastfeeding, more likely to continue breastfeeding exclusively and are slowest to discontinue any breastfeeding. In this experienced group, at 16 weeks, $52.6 \%$ recorded any breastfeeding ( $33 \%$ exclusive). In contrast, parous women with no previous breastfeeding experience are least likely to start breastfeeding with a baseline of approximately $20 \%$. In this group, at 16 weeks, only $5 \%$ were continuing with any breastfeeding ( $3.9 \%$ exclusive; table 3$)$.

\section{Prediction of stopping breastfeeding}

This analysis considered only those who initiated breastfeeding $(n=233)$ and what factors predicted cessation. As in figure 3, analyses were carried out for both exclusive breastfeeding and any breastfeeding. The final model was chosen using the AIC and the best fit model comprised the variables previous breastfeeding, intention to breastfeed, total IIFAS score and major occupational group based on ONS groups reclassified into four broad groupings. Neither age nor SIMD were included in the final model as these are strongly correlated with occupation and previous breastfeeding. Those women who initiated breastfeeding and had higher IIFAS scores were highly significantly less likely to stop breastfeeding whether 'exclusive' or 'any' breastfeeding (tables 4 and 5). Those with higher intention scores had much greater duration than those with lower intention scores and were significantly associated with lower risk of stopping 'exclusive' or 'any' breastfeeding, with a $29 \%$ and $43 \%$ lower risk, respectively.

In the final model, there was also a trend across the occupations with lower breastfeeding in routine and manual occupations. Parity was not such a strong predictor once intentions and IOWA score were included. The two most significant predictors of not stopping (for both exclusive and any breastfeeding) were high intention score and high IIFAS score (tables 4 and 5). The c-statistics for both models were $\mathrm{c}=0.649(95 \%$ CI 0.605 to 0.693$)$ and $\mathrm{c}=0.689$ (95\% CI 0.641 to 0.875 ) for 'exclusive' and 'any' breastfeeding, respectively. In these models, the IDI was the highest for the IIFAS with IDI=0.077 for 'exclusive' and IDI=0.074 for 'any' breastfeeding. In contrast, although a statistically significant predictor, the IDI was negligible for intentions from the TPB questionnaire.

\section{DISCUSSION}

As far as can be established, this is the first study of infant feeding in the weeks following birth using antenatal data gathered prospectively in real time in a large cohort. In order to achieve this, a novel method of collecting data via SMS text messaging was successfully developed, validated and utilised. This data collection method was demonstrated to have excellent reliability and validity. 24 
Table 1 Characteristics of women by feeding method at baseline delivery $(n=344)$

\begin{tabular}{|c|c|c|}
\hline & $\begin{array}{l}\text { Women who did not initiate } \\
\text { breastfeeding }(n=111) \\
{ }^{*} \text { Mean (SD) or }{ }^{* *} \%(N)\end{array}$ & $\begin{array}{l}\text { Women who initiated } \\
\text { breastfeeding }(n=233) \\
\text { Mean (SD) or } \%(N)\end{array}$ \\
\hline Gestation at baseline (weeks) ${ }^{*}$ & $32.5(5.3)$ & $31.7(5.9)$ \\
\hline Age (years) ${ }^{\star}$ & $26.6(6.2)$ & $29.6(5.4)$ \\
\hline Years since leaving school* & $15.9(2.8)$ & $17.9(1.8)$ \\
\hline \multicolumn{3}{|l|}{ Relationship status ${ }^{* *}$} \\
\hline Single & $22.5(25)$ & $5.2(12)$ \\
\hline Married & 29.7 (33) & $55.8(130)$ \\
\hline With partner & $46.8(52)$ & $38.6(90)$ \\
\hline Other & $0.0(0)$ & $0.4(1)$ \\
\hline Missing & $0.9(1)$ & $0.0(0)$ \\
\hline \multicolumn{3}{|l|}{ Living status ${ }^{\star \star}$} \\
\hline On own & $17.1(19)$ & $2.6(6)$ \\
\hline With husband or partner & $58.6(65)$ & $89.3(208)$ \\
\hline With parents & $15.3(17)$ & $5.2(12)$ \\
\hline Other & $6.3(7)$ & $1.3(3)$ \\
\hline Missing & $2.7(3)$ & $1.7(4)$ \\
\hline \multicolumn{3}{|l|}{ Parity ${ }^{\star \star}$} \\
\hline First child & $45.0(50)$ & $54.5(127)$ \\
\hline Second child & $30.6(34)$ & $29.2(68)$ \\
\hline Third child or more & $21.6(24)$ & 12.5 ( 29$)$ \\
\hline Missing & 2.7 ( 3$)$ & $3.9(9)$ \\
\hline \multicolumn{3}{|l|}{ Previous breastfeeding experience } \\
\hline Primiparous & $47.7(53)$ & $58.4(136)$ \\
\hline $\begin{array}{l}\text { Parous-no previous breastfeeding } \\
\text { experience }\end{array}$ & $43.2(48)$ & $5.2(12)$ \\
\hline Parous-previous breastfeeding experience & $9.0(10)$ & $36.5(85)$ \\
\hline Missing & $0.0(0)$ & $0.0(0)$ \\
\hline \multicolumn{3}{|l|}{ SIMD quintile** } \\
\hline Quintile 1 (most deprived) & $47.7(53)$ & $30.5(71)$ \\
\hline Quintile 2 & $20.7(23)$ & $10.3(24)$ \\
\hline Quintile 3 & $8.1(9)$ & $12.4(29)$ \\
\hline Quintile 4 & $15.3(17)$ & $30.9(72)$ \\
\hline Quintile 5 (most affluent) & $7.2(8)$ & $15.9(37)$ \\
\hline Missing & $0.9(1)$ & $0.0(0)$ \\
\hline \multicolumn{3}{|l|}{ Occupations ${ }^{* *}$} \\
\hline $\begin{array}{l}\text { Higher managerial, administrative and } \\
\text { professional occupations }\end{array}$ & $24.3(27)$ & $60.1(140)$ \\
\hline Intermediate occupations & $16.2(18)$ & $12.0(28)$ \\
\hline Routine and manual occupations & $18.9(21)$ & $11.6(27)$ \\
\hline Not in paid employment & $34.2(38)$ & $15.5(36)$ \\
\hline Missing & $6.3(7)$ & $0.9(2)$ \\
\hline Total IIFAS score & $49.8(6.29)$ & $62.8(7.46)$ \\
\hline TPB score 1: attitude to breastfeeding & $2.6(0.76)$ & $4.2(0.68)$ \\
\hline TPB score 2: subjective norm & $2.2(0.3)$ & $3.3(1.0)$ \\
\hline TPB score 3: perceived behavioural control & $2.6(0.84)$ & $3.8(0.76)$ \\
\hline TPB score 4: intention to breastfeed $†$ & $1.7(0.96)$ & $4.4(0.96)$ \\
\hline \multicolumn{3}{|c|}{ TPB score 4: intention to breastfeed categorical (\% (number) $\ddagger$} \\
\hline No breastfeeding intended & $60.4(67)$ & $3.0(7)$ \\
\hline Undecided & $38.7(43)$ & $45.1(105)$ \\
\hline Definite breastfeeding intended & $0.0(0)$ & $51.9(121)$ \\
\hline Missing & $0.9(1)$ & $0.0(0)$ \\
\hline
\end{tabular}

†On a scale of $1-5$.

$\ddagger 1$, no breastfeeding intended; $2-4$, undecided; 5 , definite breastfeeding intended.

IIFAS, lowa Infant Feeding Assessment Scale; TPB, Theory of Planned Behaviour.

A sample with a broadly similar overall SIMD profile to pregnant women in Dundee in 2009 was recruited with good representation from deprived areas which is often a problem in studying breastfeeding. Excellent follow-up through each phase of the study was achieved, and the quantitative phase was complemented by a large 
Table 2 Results of multiple logistic regression of factors associated with initiating breastfeeding $(\mathrm{n}=344)$

\begin{tabular}{|c|c|c|c|}
\hline Variable & RR (95\% Cl) & $\chi^{2}$ & p Value \\
\hline Age (years) & $1.11(1.00$ to 1.24$)$ & 4.077 & 0.044 \\
\hline \multicolumn{4}{|l|}{ Parity } \\
\hline Parous-no breastfeeding versus Primiparous & $0.28(0.12$ to 0.69$)$ & 7.798 & 0.005 \\
\hline Parous-any breastfeeding versus Primiparous & $2.67(1.15$ to 6.18$)$ & 5.294 & 0.021 \\
\hline TPB score 4: intention to breastfeed & 4.67 (2.91 to 7.49$)$ & 40.759 & $<0.0001$ \\
\hline Total IIFAS score & $1.17(1.06$ to 1.30$)$ & 9.238 & 0.002 \\
\hline \multicolumn{4}{|l|}{ Living status } \\
\hline With husband or partner versus on own & 6.07 (2.07 to 17.78$)$ & 10.806 & 0.001 \\
\hline With parents versus on own & $1.55(0.38$ to 6.27$)$ & 0.381 & 0.537 \\
\hline Other versus on own & $0.24(0.03$ to 2.27$)$ & 1.542 & 0.214 \\
\hline
\end{tabular}

amount of qualitative data gathered from a diverse sample of participants with a range of feeding experiences. $^{25}$

Our cohort's figures for breastfeeding are broadly consistent with national and local rates of breastfeeding. $68 \%$ of the sample started breastfeeding compared with local figures, $59 \%$ (local maternity database, 2009). Over the $6-8$ weeks period $29.1-33.9 \%$ were exclusively breastfeeding and 44.1-48.3\% were offering some breast milk. In comparison, Dundee City figures were exclusive $=23.3 \%$ and any $=33.4 \%$, while the exclusive breastfeeding figure at 6-8 weeks for Scotland was $26.5 \%{ }^{9}$ The generally higher rates at all time-points may be accounted for by the slightly higher numbers of women in our study from more affluent areas, while the national Infant Feeding Survey data are based on retrospective reports. ${ }^{7}$ Texting in itself may have acted as an intervention to encourage continuation of breastfeeding. It is also possible that our figures are more accurate as they are based on prospective real-time texts from the
Figure 3 (A) Time to end of exclusive breastfeeding (WHO) in all women. (B) Time to end of any breastfeeding in all women.

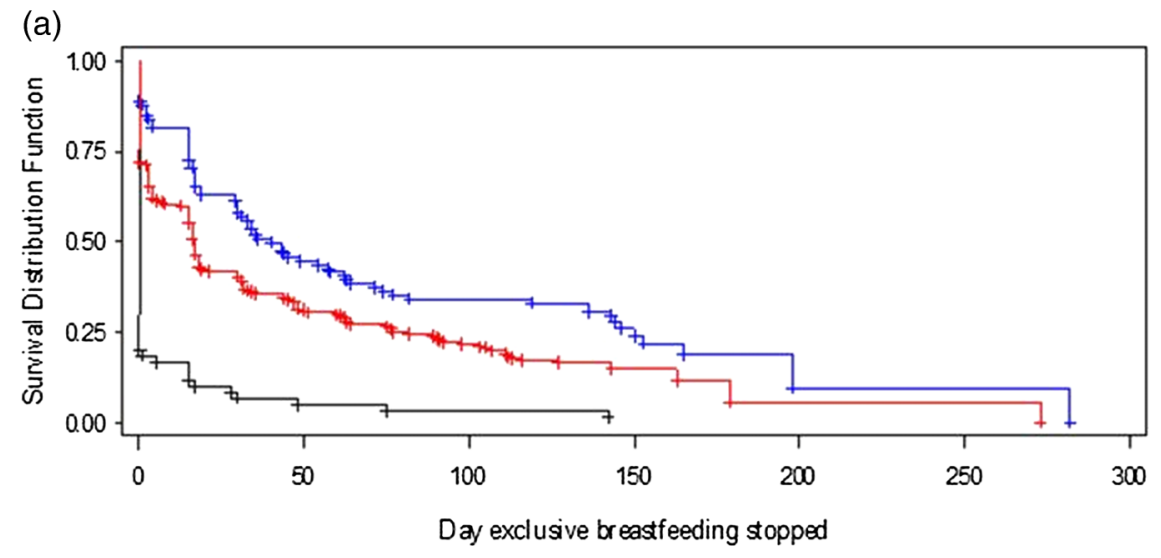

STRATA: +1 + EVER=Parous - any breasfeeding +1 EVER=Parous- no breasteeding $+1+$ EVER=Primiparous

(b)

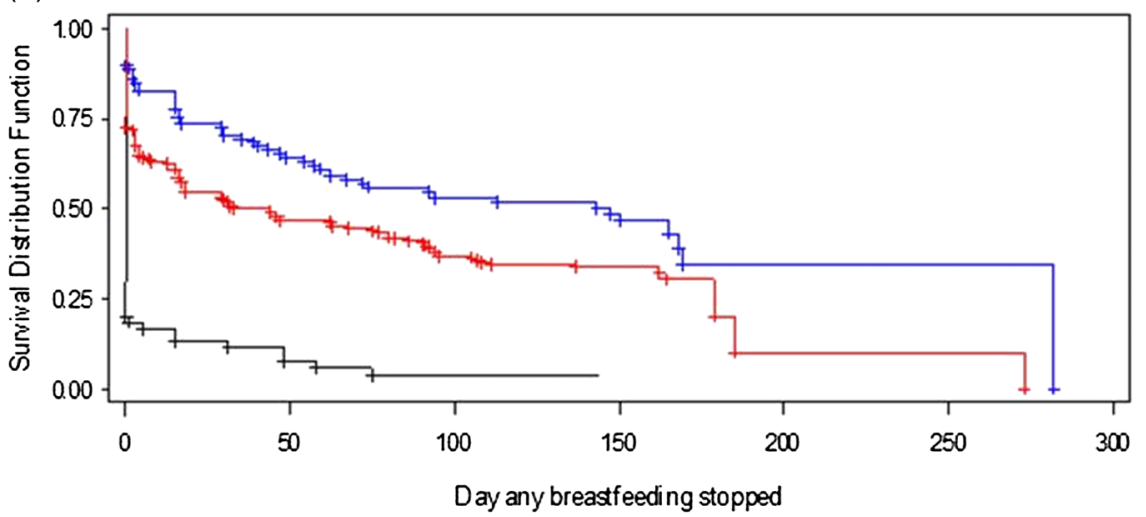

STRATA: $+1+$ EVER=Parous - any breasfeeding $\longrightarrow$ EVER=Parous- no breatfeeding EVER=Primiparous 
Table 3 Predicted breastfeeding rates at different time points according to parity and any breastfeeding of previous children (from SMS data $n=344$ )

\begin{tabular}{|c|c|c|c|c|}
\hline \multirow[b]{2}{*}{ Time } & \multicolumn{2}{|c|}{ Exclusive breastfeeding } & \multicolumn{2}{|c|}{ Any breastfeeding } \\
\hline & Per cent & $\mathrm{Cl}$ & Per cent & $\mathbf{C l}$ \\
\hline \multicolumn{5}{|l|}{ All } \\
\hline Baseline & 67.6 & (62 to 72$)$ & 68.2 & (63 to 73 ) \\
\hline 6 weeks & 33.9 & (29 to 39 ) & 48.3 & (43 to 53 ) \\
\hline 8 weeks & 29.1 & (24 to 34 ) & 44.1 & (39 to 49 ) \\
\hline 16 weeks & 20.4 & (16 to 25$)$ & 34.5 & (29 to 40$)$ \\
\hline Exit interview & 3.3 & (0 to 12$)$ & 8.5 & (1 to 27$)$ \\
\hline \multicolumn{5}{|l|}{ Primiparous } \\
\hline Baseline & 71.7 & (65 to 78$)$ & 72.3 & (65 to 78$)$ \\
\hline 6 weeks & 34.3 & (28 to 41 ) & 50.1 & (43 to 57 ) \\
\hline 8 weeks & 29.3 & (23 to 36$)$ & 46.7 & (39 to 54$)$ \\
\hline 16 weeks & 18.8 & (14 to 25$)$ & 34.5 & (28 to 41 ) \\
\hline Exit interview & 5.8 & (1 to 18$)$ & 10.1 & (1 to 32$)$ \\
\hline \multicolumn{5}{|c|}{ Parous-no previous breastfeeding } \\
\hline Baseline & 20.0 & $(11$ to 31$)$ & 18.3 & (10 to 29$)$ \\
\hline 6 weeks & 5.0 & $(1$ to 13$)$ & 11.7 & (5 to 21$)$ \\
\hline 8 weeks & 5.0 & (1 to 13$)$ & 5.8 & (2 to 14$)$ \\
\hline 16 weeks & 3.9 & (1 to 13$)$ & 5.0 & (1 to 12$)$ \\
\hline Exit interview & 1.7 & (0 to 8$)$ & 3.9 & (1 to 12$)$ \\
\hline \multicolumn{5}{|c|}{ Parous-with previous breastfeeding experience } \\
\hline Baseline & 88.8 & (81 to 94$)$ & 89.8 & (82 to 94$)$ \\
\hline 6 weeks & 46.6 & (36 to 56$)$ & 67.2 & (57 to 76$)$ \\
\hline 8 weeks & 41.4 & (32 to 51 ) & 62.0 & (52 to 71 ) \\
\hline 16 weeks & 33.0 & (24 to 42 ) & 52.6 & (42 to 62 ) \\
\hline Exit interview & 9.3 & (01 to 28) & 34.6 & (21 to 49 ) \\
\hline
\end{tabular}

Table 4 Final model using Cox regression to predict stopping 'Exclusive' breastfeeding $(n=233)$

\begin{tabular}{|c|c|c|c|}
\hline Variable & HR & $\mathbf{C l}$ & p Value \\
\hline Parous-any breastfeeding & 0.873 & (0.63 to 1.21$)$ & 0.4103 \\
\hline Parous-no breastfeeding & 0.809 & (0.41 to 1.58$)$ & 0.5367 \\
\hline Primiparous & 1.000 & & \\
\hline Higher managerial, administrative and professional occupations & 0.726 & $(0.46$ to 1.15$)$ & 0.1716 \\
\hline Intermediate occupations & 0.789 & (0.44 to 1.41$)$ & 0.4246 \\
\hline Routine and manual occupations & 0.880 & (0.50 to 1.56$)$ & 0.6601 \\
\hline Not in paid employment & 1.000 & & \\
\hline TPB score 4: intention to breastfeed (high vs low) & 0.715 & (0.53 to 0.97$)$ & 0.0317 \\
\hline Total IIFAS score (+ 10 units) & 0.553 & (0.43 to 0.71$)$ & $<0.0001$ \\
\hline
\end{tabular}

Table 5 Final model using Cox regression to predict stopping 'Any' breastfeeding $(n=233)$

\begin{tabular}{|c|c|c|c|}
\hline Variable & HR & Cl & p Value \\
\hline Parous-any breastfeeding & 0.829 & (0.56 to 1.22$)$ & 0.3426 \\
\hline Parous-no breastfeeding & 1.079 & (0.51 to 2.26$)$ & 0.8403 \\
\hline Primiparous & 1.000 & & \\
\hline Higher managerial, administrative and professional occupations & 0.602 & (0.37 to 0.99$)$ & 0.0457 \\
\hline Intermediate occupations & 0.622 & $(0.32$ to 1.21$)$ & 0.1619 \\
\hline Routine and manual occupations & 0.714 & (0.37 to 1.39$)$ & 0.3215 \\
\hline Not in paid employment & 1.000 & & \\
\hline TPB score 4: intention to breastfeed (high vs low) & 0.569 & (0.39 to 0.82$)$ & 0.0026 \\
\hline Total IIFAS score (+ 10 units) & 0.549 & (0.41 to 0.74$)$ & $<0.0001$ \\
\hline
\end{tabular}

IIFAS, lowa Infant Feeding Assessment Scale; TPB, Theory of Planned Behaviour. 
women. Overall, the consistency with known official statistics lends added validity to our results.

The mean score on the IIFAS (58.8, SD 9.36) was similar to that reported by de la Mora $e t a l^{11}$ The dichotomous nature of the 'Intentions' variable suggests that in the latter stages of pregnancy most women are clear about how they plan to feed their baby, with only a few being undecided. As in previous studies of breastfeeding using the TPB, intentions were explained by $\mathrm{PBC}$, attitudes and the IOWA score with demographic variables accounting for less of the variance. ${ }^{112829}$

The Kaplan-Meier plots show the expected pattern of breastfeeding cessation, with the steepest drop-off occurring in the first couple of weeks after birth in all women for exclusive breastfeeding. However, large differences in the duration of breastfeeding could be observed between groups. In particular, parous women with no previous breastfeeding experience stopped very quickly after birth, while primiparous women showed a similar pattern of duration to those parous women who did have previous breastfeeding experience so prior experience of breastfeeding is a strong predictor. This is similar to the findings of the recent Scottish Infant Feeding Survey data from 2010. ${ }^{7}$

In the prediction models, as others have found, ${ }^{28}$ while demographic measures were important, the measures of intention (TPB) and attitude to breastfeeding (IIFAS score) were the strongest predictors of both initiation and stopping breastfeeding. However, intention was stronger for initiating breastfeeding, while attitude was stronger for persevering with breastfeeding. This has important clinical implications. We suggest that the current interpretation of the UNICEF Baby Friendly guidelines should be revisited. Our findings indicate that a discussion with women about their intentions, in combination with an exploration of their attitude to formula feeding and breastfeeding (perhaps through use of the IIFAS) could be a powerful way of identifying those women who might need more help and support with both initiating and persevering with breastfeeding. The findings also lend weight to the targeting of younger women and women from lower socioeconomic backgrounds both for extra encouragement to breastfeed and for additional breastfeeding support. Similar to other studies that have measured PBC in pregnancy, PBC was not a significant independent predictor of breastfeeding initiation. ${ }^{28}{ }^{30}$ However, unlike McMillan et $a l,{ }^{28}$ we found that PBC was a weaker predictor of continuation than attitudes. Questions remain about the best time to measure $\mathrm{PBC}$ in relation to breastfeeding and the measures that should be used.

\section{Importance to National Health Service and possible implementation}

Breastfeeding is known to have significant short-term and long-term health benefits for both mother and infant. Increasing the number of breastfed babies through targeted interventions has the potential to prevent future ill-health, save the National Health Service money and is congruent with Government policy. ${ }^{31-33}$ The findings of this study can be used to identify women who need additional support as well as to inform the design of interventions to promote and support breastfeeding using a prediction model. Antenatal and public health interventions should aim to improve attitudes to breastfeeding generally and improve women's confidence in their ability to breastfeed. There is a need to target primigravidae during pregnancy and in the early postnatal days and weeks: as success with breastfeeding in the first pregnancy is likely to lead to more chance of feeding successfully in subsequent pregnancies. Parous women with no previous breastfeeding experience need the most support as they are most likely to give up quickly. The use of antenatal measures of intention and attitude to breastfeeding might be useful to identify women who are likely to need more support in the early days and weeks after delivery. Increased levels of support, perhaps from other women who have successfully breastfed, might be an effective intervention strategy.

\section{Future research}

This study has followed phase 1 of the MRC process, that is, the collection of initial data and determining predictors of outcome. ${ }^{23}$ The next stage will be to develop a complex intervention based on these findings both to improve rates of initiation of breastfeeding and to provide targeted support to those who start breastfeeding. In addition, the discriminative ability for initiation was excellent, but only moderate for stopping breastfeeding, while intention (TPB) was most important for initiating and attitude (IIFAS) most important for persevering with breastfeeding. This suggests that there may be further factors in stopping that could be investigated; future studies could explore this issue.

This study demonstrated the benefits of SMS messaging to collect data and so can easily be used in other studies to collect similar data. In addition, text messaging may have the potential as a cost-effective and convenient way to provide health information and support messages as part of a complex intervention. These suggestions could apply to breastfeeding, as well as having application in many other health arenas.

\section{CONCLUSIONS}

This landmark study used SMS text messaging to gather real-time data on infant feeding from birth to 16 weeks postnatal. It provides the most detailed and comprehensive data on the form and method of infant feeding. The results are consistent with Scottish national figures, hence enhancing the validity of our findings.

The construct of 'Intentions' (from the TPB) and a measure of attitude to breastfeeding (the IIFAS score) have been shown to be important in predicting future infant feeding behaviour, as well as socioeconomic 
background. Primigravidae and parous women with no previous breastfeeding experience are likely to need the most support as these groups are least likely to start breastfeeding, and most likely to stop early.

Acknowledgements We would like to thank NHS Tayside colleagues: especially the Community Midwives in Dundee, Danke McLeod, who supplied the delivery and discharge data, Child Health for information about stillbirths and neonatal deaths, Massimo Brilliante, software programmer in Health Informatics Centre (http://medicine.dundee.ac.uk/hic). We also thank women who consented to take part and who generously gave their time.

Contributors PTD designed the study, supervised the statistical analysis, drafted the final paper and approved the final version; JD designed the study, involved in design of collection tools, commented on drafts and approved the final version; AS designed the study, contributed to collection tools, commented on drafts and approved the final version; PR carried out the statistical analysis, contributed to drafts and approved the final version; EM-H involved in design of collection tools, collected data, commented on drafts and approved the final version; GK involved in design of collection tools, collected data, commented on drafts and approved the final version; JCW designed the study, supervision of the data collection, commented on drafts and approved the final version; HMW designed the study, involved in design of data collection tools, supervised the study as Pl, commented on drafts and approved the final version.

Funding Financial support for the submitted work was based on a grant from the Chief Scientist Office of Scotland (CZH/4/568)

Competing interests PTD has received research grants from Otsuka, GSK and Pfizer, provides statistical support to the Scottish Medicines Consortium, has received royalties for predictive algorithms from Arhidia Informatics; JCW has received book royalties from Springer publishing; no other relationships or activities that could appear to have influenced the submitted work.

Ethics approval The study was granted approval by the NHS Tayside Research Ethics Committee on 08.07.2009 under reference 09/S1402/28.

Provenance and peer review Not commissioned; externally peer reviewed.

Data sharing statement No additional data are available.

Open Access This is an Open Access article distributed in accordance with the Creative Commons Attribution Non Commercial (CC BY-NC 3.0) license, which permits others to distribute, remix, adapt, build upon this work noncommercially, and license their derivative works on different terms, provided the original work is properly cited and the use is non-commercial. See: http:// creativecommons.org/licenses/by-nc/3.0/

\section{REFERENCES}

1. Howie P, Forsyth J, Ogston S, et al. Protective effect of breastfeeding against infection. BMJ 1990;300:11-16.

2. Kramer MS, Kakuma R. Optimal duration of exclusive breastfeeding. Cochrane Database Syst Rev 2002;(1):CD003517.

3. Ip S, Chung M, Raman G, et al. Breastfeeding and Maternal and Infant Health Outcomes in Developed Countries Agency for Healthcare Research and Quality (US) Evidence Reports/ Technology Assessments. 2007. No. 153 http://www.ncbi.nlm.nih. gov/books/NBK38337/

4. Duijts L, Ramadhani M, Moll H. Breastfeeding protects against infectious diseases during infancy in industrialized countries. A systematic review. Matern Child Nutr 2009;5:199-210.

5. World Health Organisation. Exclusive breastfeeding for six months best for babies everywhere. 2011a. http://www.who.int/mediacentre/ news/statements/2011/breastfeeding_20110115/en/index.html

6. World Health Organisation. WHO Global Health Indicators 2011. 2011b. http://www.who.int/gho/publications/world_health_statistics/ EN_WHS2011_Part2.pdf

7. Health and Social Care Information Centre (HSCIC). Infant Feeding Survey 2010. Health and Social Care Information Centre, IFF Research. 2012. http://www.ic.nhs.uk/pubs/infantfeeding10final (accessed 23 Nov 12).

8. The Scottish Government. Better health, better care. Edinburgh: The Scottish Government, 2007.
9. ISD Scotland 2011, Breastfeeding Statistics. Information and Statistics Division. http://www.isdscotland.org/Health-Topics/ Child-Health/Publications/2011-10-25/2011-10-25-BreastfeedingSummary.pdf?40969485045 (accessed 17 May 12).

10. McInnes R, Love J, Stone D. Independent predictors of breastfeeding intention in a disadvantaged population of pregnant women. BMC Public Health 2001;1:10. http://www.biomedcentral.com/1471-2458/1/10

11. de La Mora A, Russell D, Dungy C, et al. The lowa infant feeding attitude scale: analysis of reliability and validity. J Appl Soc Psychol 1999;29:2362-80

12. Scott J, Binns C, Oddy W, et al. Predictors of breastfeeding duration: evidence from a cohort study. Pediatrics 2006;117:e646-55.

13. Scott J, Shaker I, Reid M. Parental attitudes toward breastfeeding: their association with feeding outcome at hospital discharge. Birth 2004;31:125-31.

14. Dungy C, Mclnnes R, Tappin D, et al. Infant feeding attitudes and knowledge among socioeconomically disadvantaged women in Glasgow. Matern Child Health J 2008;12:313-22.

15. Sittlington J, Stewart-Knox B, Wright M, et al. Infant-feeding attitudes of expectant mothers in Northern Ireland. Health Educ Res 2007;22:561-70.

16. Wallis $A B$, Brinzaniuc $A$, Chereches $R$, et al. Reliability and validity of the Romanian version of a scale to measure infant feeding attitudes and knowledge. Acta Paediatr 2008;97:1194-9.

17. UNICEF (nd) Baby Friendly Initiative. http://www.unicef.org.uk/ BabyFriendly/

18. Beake S, Brinzaniuc A, Chereches R, et al. A systematic review of structured compared with non-structured breastfeeding programmes to support the initiation and duration of exclusive and any breastfeeding in acute and primary health care settings. Matern Child Nutr 2012;8:141-61.

19. Kramer M, Chalmers B, Hodnett E, et al. Promotion of breastfeeding intervention trial (PROBIT). A randomized trial in the Republic of Belarus. JAMA 2001;285:413-20.

20. Tappin DM, Mackenzie J, Brown A, et al. Breastfeeding rates are increasing in Scotland. Health Bull 2001;59:102-7.

21. UNICEF. How to implement baby friendly standards: a guide for maternity settings. London: UNICEF UK Baby Friendly Initiative, 2011. http://www.unicef.org.uk/Documents/Baby_Friendly/Guidance/ Implementation\%20Guidance/Implementation_guidance_maternity_ web.pdf

22. Ajzen I. The theory of planned behavior. Organ Behav Hum Decis Processes 1991;50:179-211.

23. Campbell NC, Murray E, Darbyshire J, et al. Designing and evaluating complex interventions to improve health care. BMJ 2007;334:455-9.

24. Whitford H, Donnan $P$, Symon A, et al. Evaluating the reliability, validity, acceptability and practicality of SMS text messaging as a tool to collect research data: results from the Feeding Your Baby project. J Am Med Inform Assoc 2012;19:744-9.

25. Symon AG, Whitford H, Dalzell J. Infant feeding in Eastern Scotland: a longitudinal mixed methods evaluation of antenatal intentions and postnatal satisfaction-The Feeding Your Baby study. Midwifery 2013;29:e49-e56.

26. Standard Occupational Classification 2010 (SOC) Office for National Statistics. http://www.ons.gov.uk/ons/guide-method/classifications/ current-standard-classifications/soc2010/index.html (accessed 17 May 12).

27. Pencina MJ, D'Agostino RB Sr, D'Agostino RB Jr. Evaluating the predictive ability of a new marker: from area under the ROC curve to reclassification and beyond. Stat Med 2008;27:157-72.

28. McMillan B, Conner M, Woolridge M, et al. Predicting breastfeeding in women living in areas of economic hardship: explanatory role of the theory of planned behaviour. Psychol Health 2008;23:767-88.

29. Blyth R, Creedy D, Dennis C, et al. Effect of maternal confidence on breastfeeding duration: an application of the breastfeeding self-efficacy theory. Birth 2002;29:278-84.

30. Wambach K. Breastfeeding intention and outcome: a test of the theory of planned behavior. Res Nurs Health 1997;20:51-9.

31. The Scottish Government. A refreshed framework for maternity care in Scotland. The maternity services action group. Edinburgh: The Scottish Government, 2011a.

32. The Scottish Government. Reducing antenatal health inequalities. Outcome focused evidence into action guidance. Edinburgh: The Scottish Government, 2011b.

33. Renfrew M, Pokhrel S, Quigley M, et al. Preventing disease and saving resources: the potential contribution of increasing breastfeeding rates in the UK. UK: UNICEF, 2012. http://www.unicef. org.uk/Documents/Baby_Friendly/Research/Preventing disease saving_resources.pdf (accessed 17 May 12). 